\title{
Some extensions for the several combinatorial identities
}

\author{
Gao-Wen Xi ${ }^{1}$ and Qiu-Ming Luo ${ }^{2 *}$ (D)
}

Dedicated to Honor Professor Hari Mohan Srivastava on his 80th Birth Anniversary

"Correspondence:

luomath2007@163.com

${ }^{2}$ Department of Mathematics,

Chongqing Normal University, Chongqing Higher Education Mega

Center, Huxi Campus, Chongqing 401331, People's Republic of China

Full list of author information is

available at the end of the article

\begin{abstract}
In this paper, we give some extensions for Mortenson's identities in series with the Bell polynomial using the partial fraction decomposition. As applications, we obtain some combinatorial identities involving the harmonic numbers.
\end{abstract}

MSC: Primary 05A10; secondary 05A19; 11B65

Keywords: Combinatorial identities; Harmonic number; Bell polynomials; Partial fraction decomposition

\section{Introduction}

The higher-order harmonic numbers are defined by

$$
H_{0}^{(r)}=1 \quad \text { and } \quad H_{n}^{(r)}=\sum_{k=1}^{n} \frac{1}{k^{r}} \quad \text { for } n, r=1,2, \ldots
$$

When $n=1$, they reduce to the classical harmonic numbers $H_{n}=H_{n}^{(1)}$.

We also define the generalized higher-order harmonic numbers $H_{n}^{(r)}(z)$ as

$$
H_{0}^{(r)}(z)=1 \quad \text { and } \quad H_{n}^{(r)}(z)=\sum_{\substack{k=1 \\ k \neq-z}}^{n} \frac{1}{(k+z)^{r}}
$$

When $z=0$, they reduce to the higher-order harmonic numbers $H_{n}^{(r)}(0)=H_{n}^{(r)}$.

The standard Bell polynomials are presented in Comtet's book [5]. The modified Bell polynomials $L_{n}\left(x_{1}, x_{2}, \ldots\right)$ are defined by

$$
\exp \left(\sum_{k=1}^{\infty} x_{k} \frac{z^{k}}{k}\right)=1+\sum_{n=1}^{\infty} \mathbf{L}_{n}\left(x_{1}, x_{2}, \ldots\right) z^{n}
$$

(c) The Author(s) 2021. This article is licensed under a Creative Commons Attribution 4.0 International License, which permits use, sharing, adaptation, distribution and reproduction in any medium or format, as long as you give appropriate credit to the original author(s) and the source, provide a link to the Creative Commons licence, and indicate if changes were made. The images or other third party material in this article are included in the article's Creative Commons licence, unless indicated otherwise in a credit line to the material. If material is not included in the article's Creative Commons licence and your intended use is not permitted by statutory regulation or exceeds the permitted use, you will need to obtain permission directly from the copyright holder. To view a copy of this licence, visit http://creativecommons.org/licenses/by/4.0/. 
This expansion gives

$$
\mathbf{L}_{n}\left(x_{1}, x_{2}, \ldots\right)=\sum_{m_{1}+2 m_{2}+3 m_{3}+\cdots=n} \frac{1}{m_{1} ! m_{2} ! m_{3} ! \cdots}\left(\frac{x_{1}}{1}\right)^{m_{1}}\left(\frac{x_{2}}{2}\right)^{m_{2}}\left(\frac{x_{3}}{3}\right)^{m_{3}} \cdots .
$$

Mortenson [9, p. 990, Lemma 3.1] gave the following identities:

$$
\begin{aligned}
& \sum_{k=0}^{n}(-1)^{k}\left(\begin{array}{l}
n \\
k
\end{array}\right)\left(\begin{array}{c}
n+k \\
k
\end{array}\right) \frac{r}{r+k}=\frac{(1-r)_{n}}{(1+r)_{n}}, \quad n, r \in \mathbb{N}, \\
& \sum_{k=0}^{n}(-1)^{k}\left(\begin{array}{l}
n \\
k
\end{array}\right)\left(\begin{array}{c}
n+k \\
k
\end{array}\right)\left(H_{m+k}-H_{k}\right)=0, \quad 1 \leq m \leq n,
\end{aligned}
$$

which are called Mortenson's identities, where $(z)_{n}$ are the Pochhammer symbols defined by $(z)_{0}=1,(z)_{n}=z(z+1) \cdots(z+n-1)$.

H.M. Srivastava, J. Choi, G. Dattoli, and A. Sofo et al. investigated some infinite combinatorial series identities involving the harmonic numbers and generalized harmonic numbers by applying the hypergeometric series, Vandermonde convolutions, and Riemann zeta and polygamma functions; for details, see [1, 6-8, 10-13]. W. Chu studied some finite combinatorial identities involving the harmonic numbers by applying the partial fraction decomposition [2-4].

In this paper, we give some extensions of Mortenson's identities using the partial fraction decomposition. We obtain some new or old combinatorial identities involving the harmonic numbers and generalized harmonic numbers and propose two open problems.

\section{Extensions of Mortenson's identities}

First, we give an extension of Mortenson's identity (4).

Theorem 1 For $n \in \mathbb{N}, r \in \mathbb{N}_{0}$, and $x>0$, we have

$$
\begin{aligned}
& \sum_{k=0}^{n}(-1)^{k}\left(\begin{array}{l}
n \\
k
\end{array}\right)\left(\begin{array}{c}
n+k \\
k
\end{array}\right)\left(\frac{x}{x+k}\right)^{r} \\
& \quad=\left(\prod_{k=1}^{n} \frac{k-x}{k+x}\right)_{m_{1}+2 m_{2}+3 m_{3}+\cdots=r-1} \frac{x^{r-1}}{m_{1} ! m_{2} ! m_{3} ! \cdots}\left(\frac{U_{1}}{1}\right)^{m_{1}}\left(\frac{U_{2}}{2}\right)^{m_{2}}\left(\frac{U_{3}}{3}\right)^{m_{3}} \cdots,
\end{aligned}
$$

where $U_{k}=(-1)^{k-1} H_{n}^{(k)}(-x)+H_{n+1}^{(k)}(x-1)$.

Proof By means of the standard partial fraction decomposition we easily obtain

$$
\begin{aligned}
& \frac{(z+1)_{n}}{z(z-1) \cdots(z-n)}\left(\frac{x}{z+x}\right)^{r} \\
& \quad=\sum_{k=0}^{n}(-1)^{n-k}\left(\begin{array}{c}
n \\
k
\end{array}\right)\left(\begin{array}{c}
n+k \\
k
\end{array}\right)\left(\frac{x}{x+k}\right)^{r} \frac{1}{z-k}+\frac{\lambda}{(z+x)^{r}}+\cdots+\frac{\mu}{z+x} .
\end{aligned}
$$

Multiplying both sides of (7) by $z$ and then letting $z \rightarrow \infty$, we obtain

$$
\sum_{k=0}^{n}(-1)^{n-k}\left(\begin{array}{l}
n \\
k
\end{array}\right)\left(\begin{array}{c}
n+k \\
k
\end{array}\right)\left(\frac{x}{x+k}\right)^{r}+\mu=0 .
$$


By (2), (3), and (7) we get

$$
\begin{aligned}
\mu= & {\left[(z+x)^{-1}\right] \frac{(z+1) \cdots(z+n)}{z(z-1) \cdots(z-n)}\left(\frac{x}{z+x}\right)^{r} } \\
= & (-1)^{n+1}\left(\prod_{k=1}^{n} \frac{1}{x+k}\right)\left(\prod_{k=1}^{n}(k-x)\right) x^{r-1}\left[z^{r-1}\right] \exp \left(\sum_{k \geq 1} U_{k} \frac{z^{k}}{k}\right) \\
= & (-1)^{n+1}\left(\prod_{k=1}^{n} \frac{1}{x+k}\right)\left(\prod_{k=1}^{n}(k-x)\right) x^{r-1} \\
& \quad \times \sum_{m_{1}+2 m_{2}+3 m_{3}+\cdots=r-1} \frac{1}{m_{1} ! m_{2} ! m_{3} ! \cdots}\left(\frac{U_{1}}{1}\right)^{m_{1}}\left(\frac{U_{2}}{2}\right)^{m_{2}}\left(\frac{U_{3}}{3}\right)^{m_{3}} \cdots
\end{aligned}
$$

This completes the proof.

We next give an extension of Mortenson's identity (5) by Theorem 1.

Theorem 2 For $n, r, M \in \mathbb{N}$ and $x \geq-j, j=1,2, \ldots, M$, we have

$$
\begin{aligned}
& \sum_{k=0}^{n}(-1)^{k}\left(\begin{array}{l}
n \\
k
\end{array}\right)\left(\begin{array}{c}
n+k \\
k
\end{array}\right)\left(H_{M+k}^{(r)}(x+1)-H_{k}^{(r)}(x+1)\right) \\
& =\sum_{j=1}^{M}\left(\prod_{k=1}^{n+1} \frac{1}{x+k+j}\right)\left(\prod_{k=0}^{n-1}(k-j-x)\right) \\
& \quad \times \sum_{m_{1}+2 m_{2}+3 m_{3}+\cdots=r-1} \frac{1}{m_{1} ! m_{2} ! m_{3} ! \cdots}\left(\frac{U_{1}}{1}\right)^{m_{1}}\left(\frac{U_{2}}{2}\right)^{m_{2}}\left(\frac{U_{3}}{3}\right)^{m_{3}} \cdots
\end{aligned}
$$

where $U_{k}=(-1)^{k-1} H_{n}^{(k)}(-x-j-1)+H_{n+1}^{(k)}(x+j)$.

Proof Letting $x \longmapsto x+1$ in (6) and then letting $x \longmapsto x+j+1$, we obtain

$$
\begin{aligned}
\sum_{k=0}^{n}(-1)^{k}\left(\begin{array}{l}
n \\
k
\end{array}\right)\left(\begin{array}{c}
n+k \\
k
\end{array}\right)\left(H_{M+k}^{(r)}(x+1)-H_{k}^{(r)}(x+1)\right) \\
=\sum_{j=1}^{M} \sum_{k=0}^{n}(-1)^{k}\left(\begin{array}{l}
n \\
k
\end{array}\right)\left(\begin{array}{c}
n+k \\
k
\end{array}\right) \frac{1}{(x+k+j+1)^{r}} \\
=\sum_{j=1}^{M}\left(\prod_{k=1}^{n+1} \frac{1}{x+k+j}\right)\left(\prod_{k=0}^{n-1}(k-j-x)\right) \\
\quad \times \quad \sum_{m_{1}+2 m_{2}+3 m_{3}+\cdots=r-1} \frac{1}{m_{1} ! m_{2} ! m_{3} ! \cdots}\left(\frac{U_{1}}{1}\right)^{m_{1}}\left(\frac{U_{2}}{2}\right)^{m_{2}}\left(\frac{U_{3}}{3}\right)^{m_{3}} \cdots
\end{aligned}
$$

The proof is complete.

Taking $x=-1$ in (8), we easily obtain the following corollary. 
Corollary 3 Let $n, r, M \in \mathbb{N}$.

When $1 \leq M \leq n$, we have

$$
\begin{aligned}
& \sum_{k=0}^{n}(-1)^{k}\left(\begin{array}{l}
n \\
k
\end{array}\right)\left(\begin{array}{c}
n+k \\
k
\end{array}\right)\left(H_{M+k}^{(r)}-H_{k}^{(r)}\right)=0, \\
& \sum_{k=0}^{n}(-1)^{k}\left(\begin{array}{l}
n \\
k
\end{array}\right)\left(\begin{array}{c}
n+k \\
k
\end{array}\right)\left(H_{n+k}^{(r)}+H_{M+k}^{(r)}-2 H_{k}^{(r)}\right)=0 .
\end{aligned}
$$

When $M>n$, we have

$$
\begin{aligned}
& \sum_{k=0}^{n}(-1)^{k}\left(\begin{array}{l}
n \\
k
\end{array}\right)\left(\begin{array}{c}
n+k \\
k
\end{array}\right)\left(H_{M+k}^{(r)}-H_{k}^{(r)}\right) \\
& =\sum_{j=n+1}^{M}\left(\prod_{k=1}^{n+1} \frac{1}{k+j-1}\right)\left(\prod_{k=0}^{n-1}(k-j+1)\right) \\
& \quad \times \sum_{m_{1}+2 m_{2}+3 m_{3}+\cdots=r-1} \frac{1}{m_{1} ! m_{2} ! m_{3} ! \cdots}\left(\frac{V_{1}}{1}\right)^{m_{1}}\left(\frac{V_{2}}{2}\right)^{m_{2}}\left(\frac{V_{3}}{3}\right)^{m_{3}} \cdots,
\end{aligned}
$$

where $V_{k}=(-1)^{k-1} H_{n}^{(k)}(-j)+H_{n+1}^{(k)}(j-1)$.

\section{Some applications and two open problems}

In this section, we deduce several combinatorial identities involving the harmonic numbers from Theorems 1 and 2. We also suggest two open problems on Mortenson's identities.

Setting $r=0,1$ in (6), we obtain the familiar formulas

$$
\begin{aligned}
& \sum_{k=0}^{n}(-1)^{k}\left(\begin{array}{l}
n \\
k
\end{array}\right)\left(\begin{array}{c}
n+k \\
k
\end{array}\right)=(-1)^{n}, \\
& \sum_{k=0}^{n}(-1)^{k}\left(\begin{array}{l}
n \\
k
\end{array}\right)\left(\begin{array}{c}
n+k \\
k
\end{array}\right) \frac{x}{x+k}=\prod_{k=1}^{n} \frac{k-x}{k+x},
\end{aligned}
$$

respectively. Setting $r=1$ in Corollary 3, we obtain the following combinatorial identities involving the harmonic numbers:

$$
\begin{aligned}
& \sum_{k=0}^{n}(-1)^{k}\left(\begin{array}{l}
n \\
k
\end{array}\right)\left(\begin{array}{c}
n+k \\
k
\end{array}\right)\left(H_{M+k}-H_{k}\right)=0, \quad 1 \leq M \leq n, \\
& \sum_{k=0}^{n}(-1)^{k}\left(\begin{array}{l}
n \\
k
\end{array}\right)\left(\begin{array}{c}
n+k \\
k
\end{array}\right)\left(H_{n+k}+H_{M+k}-2 H_{k}\right)=0, \quad 1 \leq M \leq n, \\
& \sum_{k=0}^{n}(-1)^{n-k}\left(\begin{array}{l}
n \\
k
\end{array}\right)\left(\begin{array}{c}
n+k \\
k
\end{array}\right)\left(H_{M+k}-H_{k}\right)=\sum_{j=n+1}^{M} \frac{\left(\begin{array}{c}
j-1 \\
n
\end{array}\right)}{j\left(\begin{array}{c}
n+j \\
j
\end{array}\right)}, \quad M>n .
\end{aligned}
$$

Finally, we propose the following two open problems. 
Open Problem 1 For $m>n$, how do we calculate the combinatorial sums

$$
\sum_{k=0}^{n}(-1)^{k}\left(\begin{array}{l}
n \\
k
\end{array}\right)\left(\begin{array}{c}
m+k \\
k
\end{array}\right) \text { and } \sum_{k=0}^{n}(-1)^{k}\left(\begin{array}{l}
n \\
k
\end{array}\right)\left(\begin{array}{c}
m+k \\
k
\end{array}\right) \frac{x}{x+k} ?
$$

Open Problem 2 For $n \in \mathbb{N}, m, r, M \in \mathbb{N}_{0}, x>0$, what are the combinatorial sums

$$
\sum_{k=0}^{n}(-1)^{k}\left(\begin{array}{l}
n \\
k
\end{array}\right)\left(\begin{array}{c}
m+k \\
k
\end{array}\right)\left(\frac{x}{x+k}\right)^{r} \text { and } \sum_{k=0}^{n}(-1)^{k}\left(\begin{array}{l}
n \\
k
\end{array}\right)\left(\begin{array}{c}
m+k \\
k
\end{array}\right)\left(H_{M+k}^{(r)}-H_{k}^{(r)}\right) ?
$$

\section{Acknowledgements}

The authors greatly appreciate the reviewer's comments and suggestions, which improved our paper.

\section{Funding}

The present investigation was supported by Natural Science Foundation General Project of Chongqing, China under Grant cstc2019jcyj-msxmX0143.

Availability of data and materials

Not applicable.

\section{Competing interests}

The authors declare that they have no competing interests.

\section{Authors' contributions}

There was an equal amount of contributions from two authors. The authors read and approved the final manuscript.

\section{Author details}

'Department of Mathematics, Chongqing University of Science and Technology, Chongqing Higher Education Mega Center, Huxi Campus, Chongqing 401331, People's Republic of China. ${ }^{2}$ Department of Mathematics, Chongqing Normal University, Chongqing Higher Education Mega Center, Huxi Campus, Chongqing 401331, People's Republic of China.

\section{Publisher's Note}

Springer Nature remains neutral with regard to jurisdictional claims in published maps and institutional affiliations.

Received: 22 September 2020 Accepted: 8 December 2020 Published online: 09 January 2021

\section{References}

1. Choi, J., Srivastava, H.M.: Some summation formulas involving harmonic numbers and generalized harmonic numbers. Math. Comput. Model. 54, 2220-2234 (2011)

2. Chu, W.: Partial fraction decompositions and harmonic number identities. J. Comb. Math. Comb. Comput. 60 139-153 (2007)

3. Chu, W.: Summation formulae involving harmonic numbers. Filomat 26, 143-152 (2012)

4. Chu, W., Fu, A.M.: Dougall-Dixon formula and harmonic number identities. Ramanujan J. 18, 11-31 (2009)

5. Comtet, L.: Advanced Combinatorics: The Art of Finite and Infinite Expansions. Reidel, Dordrecht and Boston (1974)

6. Dattoli, G., Licciardi, S., Sabia, E., Srivastava, H.M.: Some properties and generating functions of generalized harmonic numbers. Mathematics 7, Article ID 577 (2019)

7. Dattoli, G., Srivastava, H.M.: A note on harmonic numbers, umbral calculus and generating functions. Appl. Math. Lett. 21, 686-693 (2008)

8. Gould, H.W., Srivastava, H.M.: Some combinatorial identities associated with the Vandermonde convolution. Appl. Math. Comput. 84, 97-102 (1997)

9. Mortenson, E.: Supercongruences between truncated ${ }_{2} F_{1}$ hypergeometric functions and their Gaussian analogs. Trans. Am. Math. Soc. 355, 987-1007 (2003)

10. Rassias, Th.M., Srivastava, H.M.: Some classes of infinite series associated with the Riemann zeta and polygamma functions and generalized harmonic numbers. Appl. Math. Comput. 131, 593-605 (2002)

11. Sofo, A., Srivastava, H.M.: Identities for the harmonic numbers and binomial coefficients. Ramanujan J. 25, 93-113 (2011)

12. Sofo, A., Srivastava, H.M.: A family of shifted harmonic sums. Ramanujan J. 37, 89-108 (2015)

13. Srivastava, H.M., Raina, R.K.: Some combinatorial series identities. Math. Proc. Camb. Philos. Soc. 96, 9-13 (1984) 